\title{
Visualization of the electronic phase separation in superconducting $\mathrm{K}_{x} \mathrm{Fe}_{2-y} \mathrm{Se}_{2}$
}

\author{
Yujie Chen ${ }^{1}$, Juan Jiang ${ }^{2,3}$, Haifeng Yang ${ }^{4}$, Pavel Dudin ${ }^{5}$, Alexey Barinov ${ }^{6}$, Zhongkai Liu ${ }^{4,7}$, Haihu Wen ${ }^{8}$, Lexian \\ Yang $^{1,9}(\bowtie)$, and Yulin Chen ${ }^{1,2,4,7}$ \\ ${ }^{1}$ State Key Laboratory of Low Dimensional Quantum Physics, Department of Physics, Tsinghua University, Beijing 100084, China \\ ${ }^{2}$ Department of Physics, Clarendon Laboratory, University of Oxford, Parks Road, Oxford OX1 3PU, UK \\ ${ }^{3}$ Advanced Light Source, Lawrence Berkeley National Laboratory, Berkeley, CA 94720, USA \\ ${ }^{4}$ School of Physical Science and Technology, ShanghaiTech University and CAS-Shanghai Science Research Center, Shanghai 201210, China \\ ${ }^{5}$ Synchrotron SOLEIL, L'Orme des Merisiers, Saint Aubin-BP 48, 91192 Gif sur Yvette Cedex, France \\ ${ }^{6}$ Elettra-Sincrotrone Trieste, Trieste, Basovizza 34149, Italy \\ ${ }^{7}$ ShanghaiTech Laboratory for Topological Physics, Shanghai 200031, China \\ ${ }^{8}$ National Laboratory of Solid State Microstructures and Department of Physics, Nanjing University, Nanjing 210093, China \\ ${ }^{9}$ Frontier Science Center for Quantum Information, Beijing 100084, China
}

(C) The Author(s) 2020

Received: 4 July 2020 / Revised: 3 September 2020 / Accepted: 15 September 2020

\begin{abstract}
Type-II iron-based superconductors (Fe-SCs), the alkali-metal-intercalated iron selenide $\mathrm{A}_{x} \mathrm{Fe}_{2-y} \mathrm{Se}_{2}(\mathrm{~A}=\mathrm{K}, \mathrm{TI}, \mathrm{Rb}$, etc.) with a superconducting transition temperature of $32 \mathrm{~K}$, exhibit unique properties such as high Néel temperature, Fe-vacancies ordering, antiferromagnetically ordered insulating state in the phase diagram, and mesoscopic phase separation in the superconducting materials. In particular, the electronic and structural phase separation in these systems has attracted intensive attention since it provides a platform to unveil the insulating parent phase of type-II Fe-SCs that mimics the Mott parent phase in cuprates. In this work, we use spatial- and angle-resolved photoemission spectroscopy to study the electronic structure of superconducting $\mathrm{K}_{x} \mathrm{Fe}_{2-y} \mathrm{Se}_{2}$. We observe clear electronic phase separation of $\mathrm{K}_{x} \mathrm{Fe}_{2-y} \mathrm{Se}_{2}$ into metallic islands and insulating matrix, showing different $\mathrm{K}$ and $\mathrm{Fe}$ concentrations. While the metallic islands show strongly dispersive bands near the Fermi level, the insulating phase shows an energy gap up to $700 \mathrm{meV}$ and a nearly flat band around $700 \mathrm{meV}$ below the Fermi energy, consistent with previous experimental and theoretical results on the superconducting $\mathrm{K}_{1-x} \mathrm{Fe}_{2} \mathrm{Se}_{2}$ (122 phase) and Fe-vacancy ordered $\mathrm{K}_{0.8} \mathrm{Fe}_{1.6} \mathrm{Se}_{2}(245$ phase), respectively. Our results not only provide important insights into the mysterious composition of phase-separated superconducting and insulating phases of $\mathrm{K}_{x} \mathrm{Fe}_{2-y} \mathrm{Se}_{2}$, but also present their intrinsic electronic structures, which will shed light on the comprehension of the unique physics in type-II Fe-SCs.
\end{abstract}

\section{KEYWORDS}

spatial- and angle-resolved photoemission spectroscopy ( $\mu$-ARPES), iron-based superconductors, phase separation, electronic structure

\section{Introduction}

Ever since the discovery of superconductivity at $32 \mathrm{~K}$ in the type-II iron-based superconductors (Fe-SCs), $\mathrm{A}_{x} \mathrm{Fe}_{2-y} \mathrm{Se}_{2}$ $(\mathrm{A}=\mathrm{K}, \mathrm{Tl}, \mathrm{Rb}$, etc.), this unique family of Fe-SCs has evoked considerable research interests due to its novel physical properties, including extremely high Néel temperature, large magnetic moment of $\mathrm{Fe}$ atoms, unique electronic structure, interesting phase diagram, and mesoscopic phase separation [1-10]. The absence of hole pockets near the $\Gamma$ point in the Fermi surface (FS) of $\mathrm{A}_{x} \mathrm{Fe}_{2-y} \mathrm{Se}_{2}$ excludes the $\mathrm{s} \pm$ pairing symmetry in this system, which is widely accepted as a prerequisite for the superconductivity in Fe-SCs [11-13]. In the phase diagram of $\mathrm{A}_{x} \mathrm{Fe}_{2-y} \mathrm{Se}_{2}$, there exists an antiferromagnetically (AFM) ordered insulating state that mimics the parental Mott insulating state in the cuprate high temperature superconductors $[9,14,15]$. Moreover, the superconducting $\mathrm{A}_{x} \mathrm{Fe}_{2-y} \mathrm{Se}_{2}$ is shown to be ubiquitously phase separated into superconducting islands and insulating background [3, 10, 16-18]. The major insulating phase crystallizes in a block-spin AFM phase with a composition close to $\mathrm{A}_{0.8} \mathrm{Fe}_{1.6} \mathrm{Se}_{2}$ (245 phase), which shows Fe-vacancy-ordering at low temperatures [5, 19-22], while the minor metallic phase, i.e., the superconducting component, shows a composition close to $\mathrm{A}_{1-x} \mathrm{Fe}_{2} \mathrm{Se}_{2}$ (122 phase). Various models have been proposed for the accurate structure of the superconducting 122 phase, including $(\sqrt{2} \times \sqrt{5})$ charge ordering, rhombus iron vacancy, alkali intercalated $\mathrm{FeSe}$ in an orthorhombic $\mathrm{BaFe}_{2} \mathrm{As}_{2}$-like structure, and $(\sqrt{8} \times \sqrt{10})$ parallelogram structure with Fe vacancy $[10,19,20,23,24]$, which are still under heavy debate. Based on these experimental and theoretical results, different scenarios of the parent phase of type-II Fe-SC have been proposed, including Mott insulator [25-27], the $(\sqrt{5} \times \sqrt{5})$ Fe-vacancy ordered 245 phase $[9,14$, 15], a semiconducting phase [28], and a $\mathrm{A}_{2} \mathrm{Fe}_{7} \mathrm{Se}_{8}$ phase with 
$(\sqrt{8} \times \sqrt{10})$ Fe-vacancy ordering as the possible parent phase [10].

While the structural phase separation in $\mathrm{A}_{x} \mathrm{Fe}_{2-y} \mathrm{Se}_{2}$ has been extensively studied by various spatial-resolved techniques such as scanning tunnelling microscopy (STM), scanning electron microscope (SEM), transmission electron microscope (TEM) and nanofocused X-ray diffraction (XRD) [10, 16-19], the intrinsic electronic structures of the phase-separated 122 and 245 phases are not well understood yet. Angle-resolved photoemission spectroscopy (ARPES) has revealed the abnormal FS topology, isotropic superconducting gap and orbital-selective Mott phase in $\mathrm{A}_{x} \mathrm{Fe}_{2-y} \mathrm{Se}_{2}[11-13,29-32]$. However, due to the lack of spatial resolution, the conventional ARPES measures the mixture of the electronic structures of different phases of $\mathrm{A}_{x} \mathrm{Fe}_{2-y} \mathrm{Se}_{2}$. Direct measurements on the intrinsic electronic structures of the phase-separated 122 and 245 phases are necessary in order to understand the unique properties of $\mathrm{A}_{x} \mathrm{Fe}_{2-y} \mathrm{Se}_{2}$. The realization of high spatial-resolution in ARPES by adopting micro-focused beam spot (spatial-resolved ARPES or $\mu$-ARPES) provides an ideal tool to investigate the electronic phase separation and related fine local electronic structures of different phases in $\mathrm{A}_{x} \mathrm{Fe}_{2-y} \mathrm{Se}_{2}$. Previous $\mu$-ARPES measurements have revealed interconnected networks of the minor 122 phase on the background matrix of 245 insulating phase [21, 33, 34], consistent with other local structural and magnetic measurements. However, partially due to the limited energy and spatial resolutions, the subtle microstructure of the minor 122 phase was not revealed and the investigation on the detailed electronic structures of the 122 and 245 phases is still essentially lacking.

In this manuscript, we use $\mu$-ARPES to investigate the intrinsic electronic structure of the phase-separated $\mathrm{K}_{x} \mathrm{Fe}_{2-y} \mathrm{Se}_{2}$. We show that the two phases are separated into 122 phase islands of micrometer scale and an insulating matrix of 245 phase. The characteristic K 3p peak in the core-level spectra shows distinctive intensity in the 122 and 245 regions, respectively; while the Se 3d peak shows a chemical shift of about $400 \mathrm{meV}$ and a change of the line shape, suggesting different chemical environment for the Se atoms in the two phases. Near the Fermi energy $\left(E_{\mathrm{F}}\right)$, the measured electronic structure of the 122 phase resembles the result of conventional ARPES measurements. However, a nearly flat band at $\sim 700 \mathrm{meV}$ below $E_{\mathrm{F}}$ in the conventional ARPES measurements is absent in our $\mu$-ARPES measurements on the 122 phase, and appears in the insulating 245 phase. The observed band gap of the insulating 245 phase is as large as $700 \mathrm{meV}$, consistent with ab-initio calculation on the 245 phase with block-spin AFM ordering $[35,36]$. Our results unveil the intrinsic electronic structures of the phase-separated 122 and 245 phases, which will help to understand the puzzling superconducting transition in $\mathrm{K}_{x} \mathrm{Fe}_{2-y} \mathrm{Se}_{2}$ and the parent compound of this intriguing type of superconductor. Our experiments also illustrate $\mu$-ARPES as a powerful tool to directly investigate the electronic phaseseparation phenomena in inhomogeneous materials with both high spatial and momentum resolutions.

\section{Results and discussion}

$\mathrm{K}_{1-x} \mathrm{Fe}_{2} \mathrm{Se}_{2}$ (122 phase) crystallizes in a $122 \mathrm{ThCr}_{2} \mathrm{Si}_{2}$ structure, similar to the iron pnictide $\mathrm{BaFe}_{2} \mathrm{As}_{2}$ as shown in Fig. 1(a). It can be viewed as alkali intercalated alpha-FeSe [1]. The insulating 245 phase crystallizes in a similarly layered structure (Fig. 1(b)), except that there are additional Fe vacancies every fifth $\mathrm{Fe}$ atoms [4]. At low temperature, the $\mathrm{Fe}$ vacancies are proposed to form a $(\sqrt{5} \times \sqrt{5})$ ordering $[5,19,20]$.

$\mu$-ARPES, combing both spatial and momentum resolutions, promises a direct investigation of the intrinsic electronic structure of phase-separated materials as schematically demonstrated in Figs. 1(c) and 1(d). The light beam needs to be strongly focused in order to get high enough spatial resolution. Usually, a Fresnel zone plate or a Schwarzschild mirror is used to focus the beam down to less than 1 micron. In order to make the samples with larger size of the superconducting islands for better $\mu$-ARPES observation, we used the slow furnace cooling (SFC) method

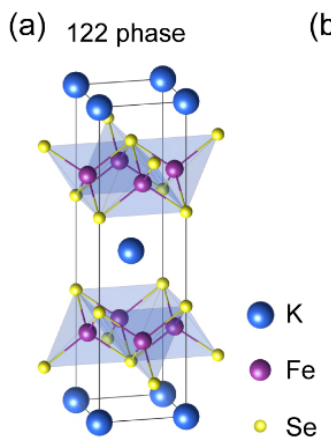

(b)

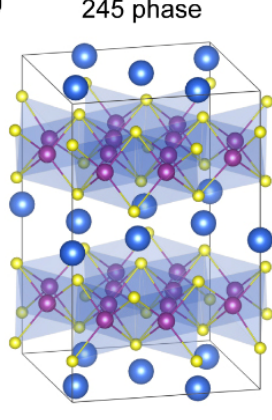

(c)

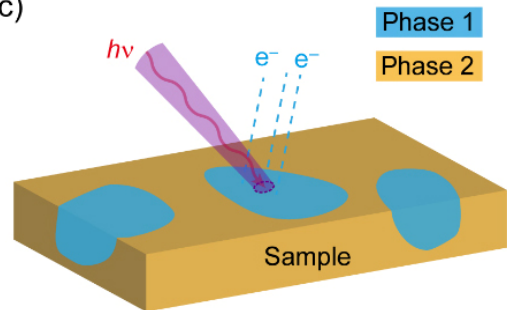

(d)

(e)
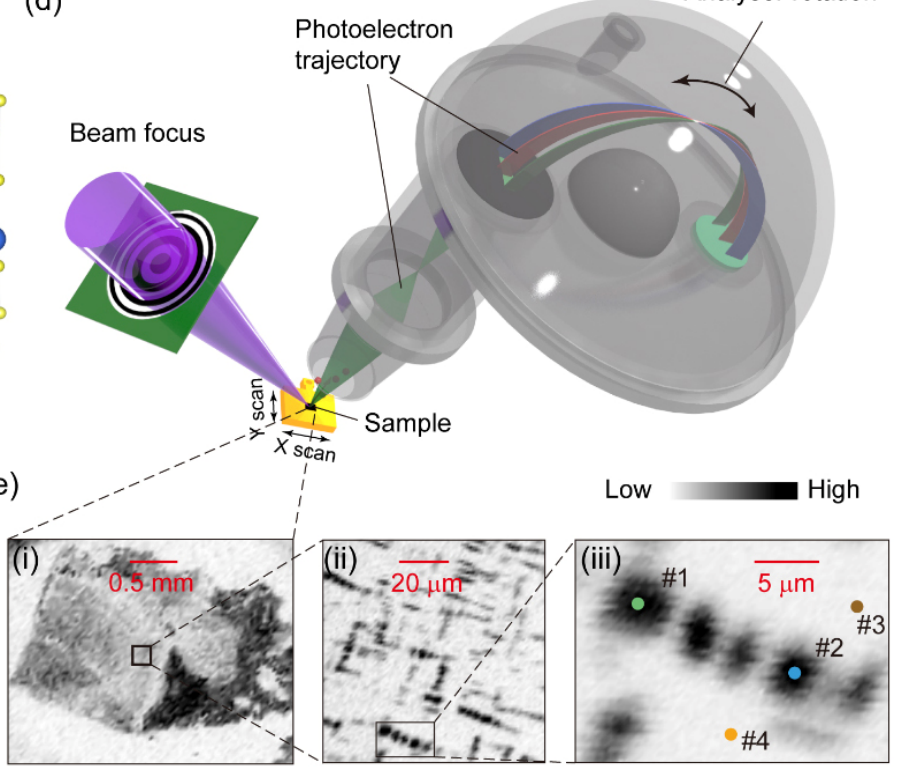

Figure 1 Crystal structure of separated phases in $\mathrm{K}_{x} \mathrm{Fe}_{2-y} \mathrm{Se}_{2}$ and the schematic illustration of $\mu$-ARPES. (a) The crystal structure of superconducting $\mathrm{K}_{1-x} \mathrm{Fe}_{2} \mathrm{Se}_{2}$ (122 phase). (b) The crystal structure of the insulating $\mathrm{K}_{0.8} \mathrm{Fe}_{1.6} \mathrm{Se}_{2}$ (245 phase). (c) With strongly focused beam, the electrons can be excited from different phases in a phase-separated material. (d) A cartoon of $\mu$-ARPES setup. The light beam can be focused down to sub-micron size to ensure high spatial resolution. By scanning the sample position in real space, the local electronic structures at selected sample positions can be measured. To measure the electrons emitted from different emission angle at a fixed sample position, the electron analyzer instead of the sample rotates, is indicated by the black arrow. (e) Real-space photoemission intensity map of cleaved $\mathrm{K}_{x} \mathrm{Fe}_{2-y} \mathrm{Se}_{2}$ sample measured by $\mu$-ARPES. 
to treat the samples, in this case some of the islands can grow up and have dimensions as big as a couple of micrometers [10]. The onset value of the superconducting transition temperature $\left(T_{c}\right)$ is around $25 \mathrm{~K}$ in these SFC treated samples. In comparison with normally treated samples with $T_{\mathrm{c}} \sim 31 \mathrm{~K}$, the degraded $T_{\mathrm{c}}$ value in our samples is most likely due to a small difference in the precise chemical composition of the superconducting 122 phase or may possibly be associated with a change in the electronic interaction of the two phases (increased size of superconducting domains and decreased amount of interface), which may have potential influences on the measured electronic structures. From our SEM and XRD measurements, the chemical composition of the superconducting area is about $\mathrm{K}_{0.64} \mathrm{Fe}_{1.78} \mathrm{Se}_{2}$, which is close to the composition of normally treated sample and the related crystal structure doesn't change [10]. By scanning in the real space, the distinctive electronic structures of different phases in $\mathrm{K}_{x} \mathrm{Fe}_{2-y} \mathrm{Se}_{2}$ can be measured at selected sample positions (Fig. 1(c)). Figure 1(e)(i) shows the real-space ARPES intensity map near $E_{\mathrm{F}}$ scanning across the whole sample surface, showing the basic topography of cleaved $\mathrm{K}_{x} \mathrm{Fe}_{2-y} \mathrm{Se}_{2}$. The zoom-in plot in Fig. 1(e)(ii) shows dark regions with higher intensity forming networks with typical width of $0.5-$ $1 \mu \mathrm{m}$, confirming the existence of electronic phase separation. The further zoom-in plot in Fig. 1(e)(iii) shows discontinuous islands, consistent with previous local structural and magnetic measurements [5, 10, 19-22].

Figure 2 shows the real-space variation of the core-level photoemission spectra, which can provide important insights into the related chemical composition. The electronic phase separation can be well resolved in an energy window of at least $2.5 \mathrm{eV}$ below $E_{\mathrm{F}}$, where the $\mathrm{Fe} 3 \mathrm{~d}$ orbitals dominate the density of states (Fig. 2(a)). We select 2 points from each phase (marked in Figs. 1(e) and 2(d)(left)) to show the spatial variation of the core-level spectra. As shown in Fig. 2(b) and zoom-in plots in Fig. 2(c), the identical spectra from different locations of the same phase suggest an intrinsic phase separation instead of randomly inhomogeneous variation of the chemical composition. From the spectra near $E_{\mathrm{F}}$ shown in Fig. 2(c)(i), we observe much higher density of states of the Fe $3 \mathrm{~d}$ orbitals on the dark islands, while a large energy gap exists in the major bright background. Therefore, we attribute the dark islands to the conductive 122 phase and the bright background to the insulating 245 phase with Fe vacancies [21, 33, 34]. Although the observed metallic islands show discontinuous pattern on the cleaved surface of bulk $\mathrm{K}_{x} \mathrm{Fe}_{2-y} \mathrm{Se}_{2}$, we should note that these separated islands are interconnected in real space accounting for the bulk superconductivity of the sample [34].

In addition to the difference of the photoemission intensity near $E_{\mathrm{F}}$, we also observe differences near the characteristic K $3 \mathrm{p}$ and Se 3d peaks, as shown in Figs. 2(c)(ii) and 2(c)(iii). Near the Se 3d peaks, the 122 phase shows a main peak near $54.7 \mathrm{eV}$ and a shoulder near $55.5 \mathrm{eV}$, while the 245 phase shows double peaks located near 54.3 and $55.3 \mathrm{eV}$. The difference in the line shape of Se 3d peaks is attributed to a chemical shift due to the different chemical environments in the two phases (Figs. 1(a) and 1(b)). We emphasize that the overall intensities of Se $3 \mathrm{~d}$ peaks in the two phases are quite similar, suggesting a nearly uniform distribution of Se atoms in the 122 and 245 phases. Near the K 3p peak, in contrast, a clear difference of the peak intensity is observed, suggesting a lower K concentration in the 122 phase than in the 245 phase. Such photoemission results are in good agreement with the SEM experiments on the same samples [10]. For Fe 3p core level $(\sim 52.7 \mathrm{eV})$, possibly due to the poor photoemission cross-section under the photon energy used here $(h v=74 \mathrm{eV})$, we do not observe significant contribution from Fe $3 p$ orbitals in the core-level spectra. The (a)

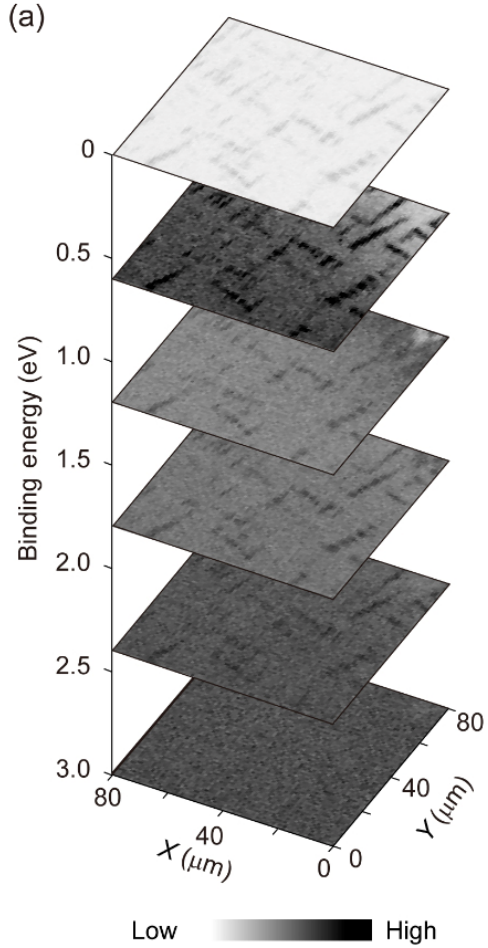

(b)

(c)
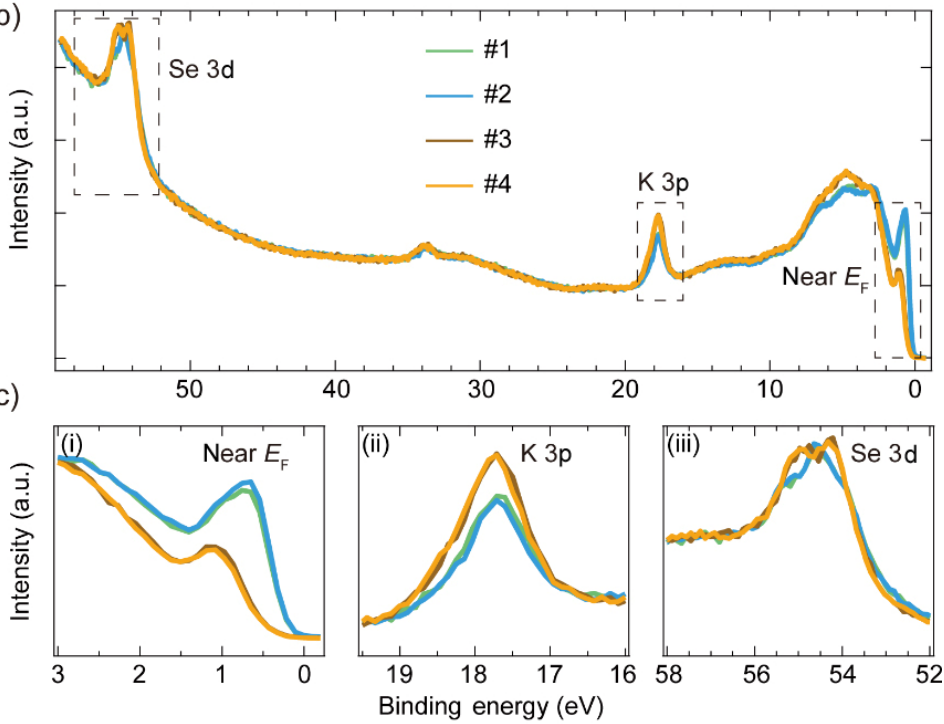

(d)
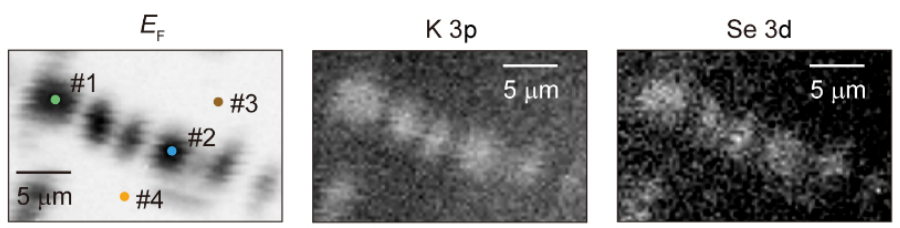

Figure 2 Phase separation in $\mathrm{K}_{x} \mathrm{Fe}_{2-y} \mathrm{Se}_{2}$ measured by $\mu$-ARPES. (a) Stacking plot of the $\mu$-ARPES intensity map in real space. The dark network-like islands are attributed to the 122 phase with a higher density of states near the Fermi energy $\left(E_{\mathrm{F}}\right)$. (b) The core-level spectra collected at different sample positions corresponding to the 122 and 245 phases. Two points are selected for each phase, where points \#1 and \#2 refer to the 122 phase and points \#3 and \#4 refer to the 245 phase, as marked in Fig. 1(e)(iii). (c) The enlarged core-level spectra near the $E_{\mathrm{F}}$ (i), K 3p peak (ii), and Se $3 \mathrm{~d}$ peak (iii). (d) The phase separation characterized by the real space photoemission intensity map at different binding energies corresponding to $E_{\mathrm{F}}(\mathrm{left}), \mathrm{K} 3 \mathrm{p}$ peak (middle) and Se $3 \mathrm{~d}$ peak (right). The sample points \#1-\#4 indicate the positions where the spectra in (b) are collected. 
spatial mapping of the photoemission intensity near $E_{\mathrm{F}}, \mathrm{K} 3 \mathrm{p}$, and Se 3d peaks are summarized in Fig. 2(d), which reflects the electronic phase separation at characteristic binding energies.

In Fig. 3, we present a close examination of the band dispersion near $E_{\mathrm{F}}$ of both 122 and 245 phases. In the 122 phase, we observe hole-like band dispersion together with a weak electron pocket near $E_{\mathrm{F}}$ at the $\Gamma$ point and an electron-like band dispersion at the M point (Figs. 3(a) and 3(b)), consistent with previous studies $[33,34]$. Figure 3 (c) schematically plots the major band dispersions of the 122 phase, which is in good consistence with previous ARPES measurements [11-13, 29-31]. As an important fact in understanding the pairing symmetry of superconducting in Fe-SCs, we confirm the absence of the hole pocket in the FS of the intrinsic 122 phase [11-13]. In the insulating 245 phase, on the other hand, we observe an energy gap as large as $700 \mathrm{meV}$ (Figs. 3(d) and 3(e)) and a weakly dispersive band near $700 \mathrm{meV}$ below $E_{\mathrm{F}}$ with strong spectral weight, as schematically shown in Fig. 3(f). Figure 3(g) compares the energy distribution curves (EDCs) at different momentum positions collected on different phases, showing the drastically distinctive line shapes. Clearly, the band dispersion of the 122 phase cannot be reproduced by a simple rigid shift of the band structure of the insulating 245 phase, indicating the fundamentally different electronic structures of the two phases.

In Fig. 4, we compare the electronic structures of the 122 phase studied with $\mu$-ARPES and conventional ARPES (the same setup with $\mu$-ARPES except that the beam is defocused). The measured band structures are similar to each other near $E_{\mathrm{F}}$ (Figs. 4(a) and 4(b)). However, a broad and nearly disperseless band only appears in the result measured with defocused beam that covers both 122 and 245 phases, which can be nicely reproduced by artificially summing up the results measured on the 122 and 245 phases with $\mu$-ARPES (Figs. 4(b) and 4(c)), as further proved by the EDCs near $\Gamma$ and $M$ points in Fig. 4(d). Our measurement suggests that the measurement of the electronic structure of the 122 phase using conventional ARPES is not strongly affected by the insulating background.

With high-quality $\mu$-ARPES data, we have visualized the intrinsic electronic structures of phase-separated 122 and 245 phases of $\mathrm{K}_{x} \mathrm{Fe}_{2-y} \mathrm{Se}_{2}$. The electronic states in the 122 and 245
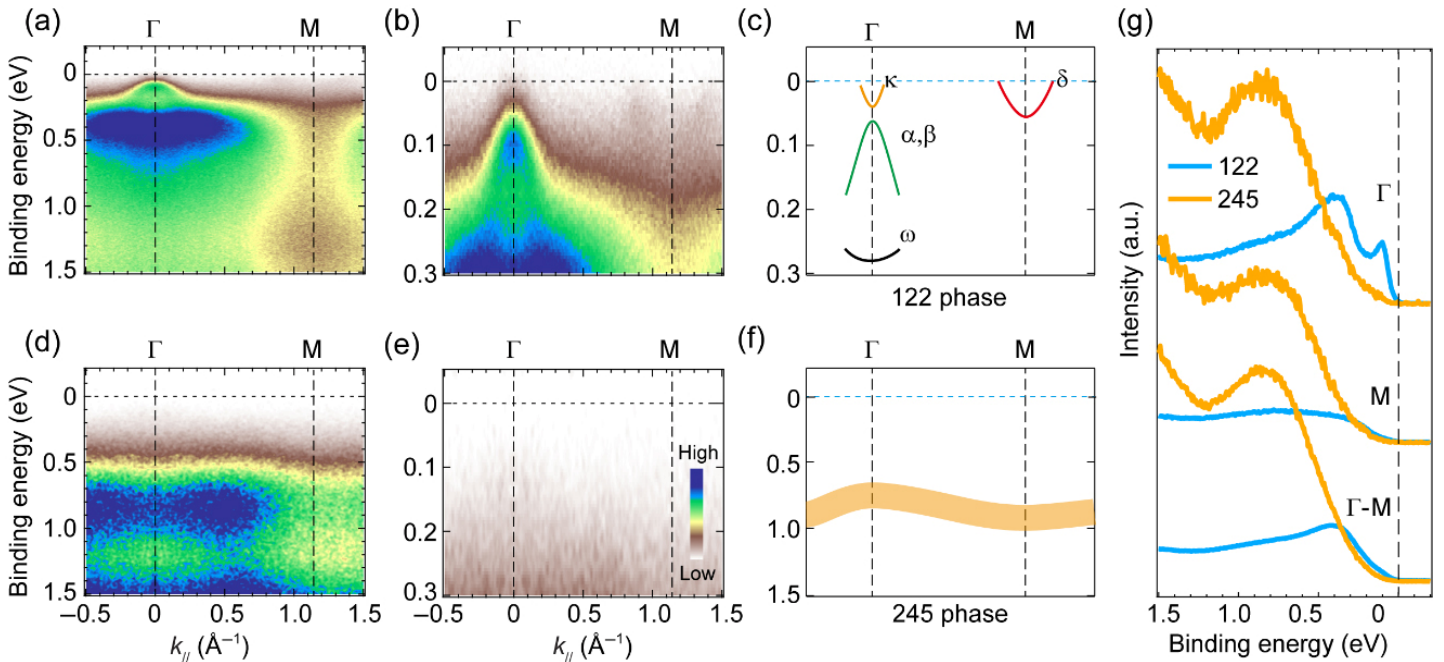

Figure 3 The comparison of the electronic structures of the metallic 122 and insulating 245 phases. (a) and (b) Band dispersion of 122 phase along $\Gamma-\mathrm{M}$ in different energy windows. (c) Schematic plots of band dispersion measured near $E_{\mathrm{F}}$. (d) and (e) Band dispersion of insulating 245 phase along $\Gamma-\mathrm{M}$ in different energy windows. (f) Schematic plot of band dispersion of insulating 245 phase. (g) Comparison of the energy distribution curves (EDCs) of two phases near the $\Gamma$ and $M$ points as well as integrated along $\Gamma-\mathrm{M}$.

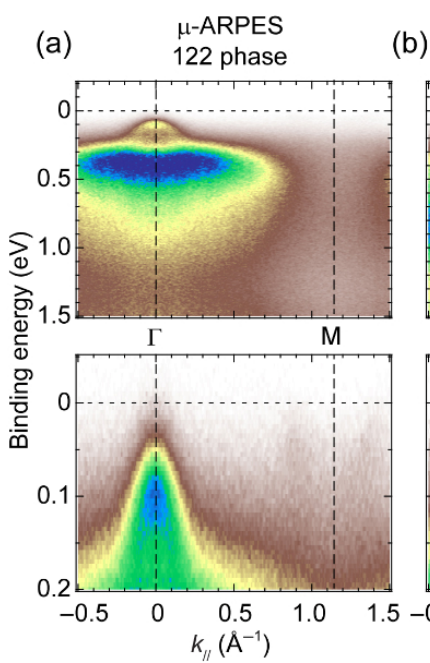

(b) Defocused $\mu$-ARPES

$\mu-A R P E S$
(c) $\quad 122+245$ phase
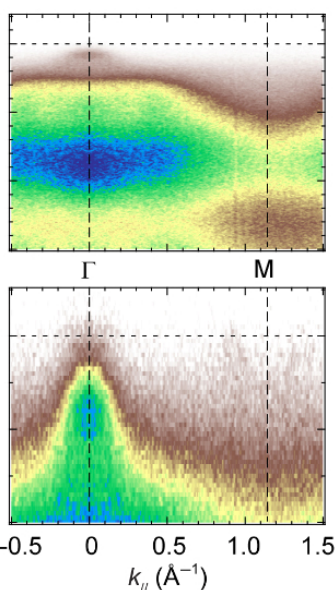
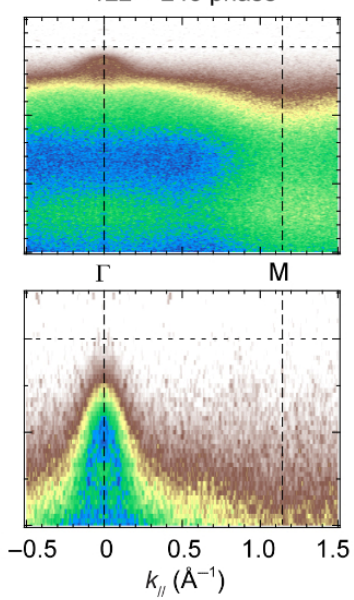

(d)

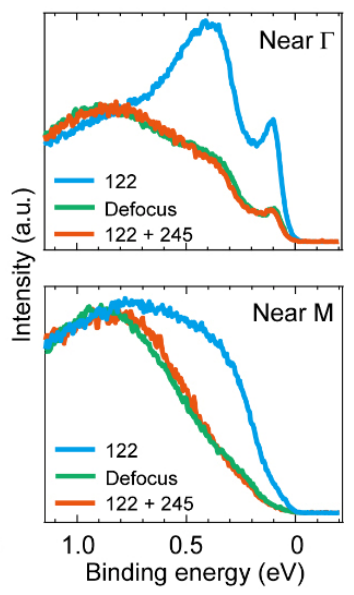

Figure 4 The comparison of the electronic structures of the 122 phase obtained with $\mu$-ARPES and conventional ARPES. (a) The intrinsic electronic structure of the 122 phase obtained with $\mu$-ARPES. (b) The electronic structure obtained by conventional ARPES using the same setup with $\mu$-ARPES except the beam is defocused. (c) ARPES intensity map obtained by artificially summing up ARPES spectra from the 122 and insulating 245 phases measured with $\mu$-ARPES. (d) Comparison of EDCs near the $\Gamma$ and $M$ points measured with $\mu$-ARPES and conventional ARPES (or defocused $\mu$-ARPES). 
phases are well separated in energy space, which guarantees that the conventional high-resolution ARPES reveals the correct band structure of superconducting $\mathrm{K}_{x} \mathrm{Fe}_{2-y} \mathrm{Se}_{2}$ near $E_{\mathrm{F}}$, confirming the absence of the hole pockets around the $\Gamma$ point in the FS. However, the EDC line shape is greatly changed by the mixture of the signals from 122 and 245 phases (Fig. 4(d)). Therefore, it is important to measure the intrinsic electronic structure of the 122 phase using $\mu$-ARPES. The dispersions at high binding energies (>500 meV), which are likewise important for accurately understanding the intrinsic electronic structure of superconducting 122 phase, are strongly contaminated by the electronic structure of the insulating 245 phase and can only be measured by $\mu$-ARPES. On the other hand, it is worth to emphasize that the measured electronic structure of the insulating 245 phase, including the energy gap of $\sim 700 \mathrm{meV}$ and the nearly disperseless band, shows good consistence with ab-initio calculation on the 245 phase with block-spin AFM ordering [35, 36] and optical measurement [37], which is important for understanding the parental phase of type-II Fe-SCs. Finally, the self-aggregation of the 122 and 245 phases with drastically different electronic structures at low temperature suggests subtle interplay between the two phases. For example, with the increased size of the superconducting island, the $T_{c}$ value decreases [10], which may be due to the decreased amount of interface. Other effects, such as the balance between the chemical potentials, the proximity effect, and the strain effect should be taken into account as well in understanding the unique electronic properties of $\mathrm{K}_{x} \mathrm{Fe}_{2-y} \mathrm{Se}_{2}$.

\section{Conclusion}

In conclusion, we have clearly revealed the electronic phase separation in type-II Fe-SC $\mathrm{K}_{x} \mathrm{Fe}_{2-y} \mathrm{Se}_{2}$ by means of spatialresolved ARPES. Firstly, our $\mu$-ARPES measurements unveil the intrinsic electronic structure of the superconducting 122 phase, which compellingly confirms the unusual FS topology of superconducting $\mathrm{K}_{x} \mathrm{Fe}_{2-y} \mathrm{Se}_{2}$. Secondly, we provide important insights into the composition of the phase separated 122 and 245 phases. Thirdly, we directly visualize the electronic structure and band gap of the insulating 245 phase, which is important for further improvements in constructing theoretical models for phase-separated $\mathrm{K}_{x} \mathrm{Fe}_{2-y} \mathrm{Se}_{2}$. Our results thus shed light on the understanding of the unique electronic properties of $\mathrm{K}_{x} \mathrm{Fe}_{2-y} \mathrm{Se}_{2}$ and we emphasize that the interplay between the 122 and 245 phases may play an important role in the superconductivity of $\mathrm{K}_{x} \mathrm{Fe}_{2-y} \mathrm{Se}_{2}$.

\section{Materials and methods}

\subsection{Materials}

$\mathrm{K}_{x} \mathrm{Fe}_{2-y} \mathrm{Se}_{2}$ single crystals were prepared by self-flux method. $\mathrm{K}$ pieces and FeSe powders were used as precursors. They were mixed in an atomic ratio of $\mathrm{K}:(\mathrm{FeSe})=0.8: 2$, and then the mixture was loaded into an alumina crucible and sealed in a quartz tube under vacuum. The mixture was subsequently heated up to $1,030^{\circ} \mathrm{C}$ and held for $3 \mathrm{~h}$. The sample was cooled down to $800{ }^{\circ} \mathrm{C}$ at a rate of $4{ }^{\circ} \mathrm{C} / \mathrm{h}$, and then it was cooled to room temperature by switching off the power of the furnace. All the weighing, mixing, grinding and pressing procedures were finished in a glove box under argon atmosphere with the oxygen and moisture below 0.1 p.p.m.

\subsection{Spatial-resolved ARPES measurements}

$\mu$-ARPES measurements were carried out at the Spectromicroscopy beamline 3.2L at Elettra-Sincrotrone in Trieste and ANTARES beamline at Soleil Synchrotron facility. The samples were cleaved in-situ under ultra-high vacuum with pressure below $2 \times 10^{-10} \mathrm{mbar}$ and measured below $25 \mathrm{~K}$. Data were collected with a non-commercial electron analyser (Scienta R4000) with overall energy, angle, and spatial resolutions of $50 \mathrm{meV}(20 \mathrm{meV}), 0.5^{\circ}\left(0.2^{\circ}\right)$, and $\sim 1$ micron $(\sim 120 \mathrm{~nm})$ at Spectromicroscopy (ANTARES) beamline, respectively.

\section{Acknowledgments}

This work was supported by the National Natural Science Foundation of China (No. 11427903, 11774109 and 11674229), the National Key R\&D Program of China (Nos. 2017YFA0304600 and 2017YFA0305400), and EPSRC Platform Grant (No. EP/ M020517/1). L. X. Y. acknowledges the support from Tsinghua University Initiative Scientific Research Program.

Open Access This article is licensed under a Creative Commons Attribution 4.0 International License, which permits use, sharing, adaptation, distribution and reproduction in any medium or format, as long as you give appropriate credit to the original author(s) and the source, provide a link to the Creative Commons licence, and indicate if changes were made.

The images or other third party material in this article are included in the article's Creative Commons licence, unless indicated otherwise in a credit line to the material. If material is not included in the article's Creative Commons licence and your intended use is not permitted by statutory regulation or exceeds the permitted use, you will need to obtain permission directly from the copyright holder.

To view a copy of this licence, visit http://creativecommons.org/licenses/by/4.0/.

\section{References}

[1] Guo, J. G.; Jin, S. F.; Wang, G.; Wang, S. C.; Zhu, K. X.; Zhou, T. T.; $\mathrm{He}, \mathrm{M}$.; Chen, X. L. Superconductivity in the iron selenide $\mathrm{K}_{x} \mathrm{Fe}_{2} \mathrm{Se}_{2}$ $(0 \leq x \leq 1.0)$. Phys. Rev. B 2010, 82, 180520(R).

[2] Ying, J. J.; Wang, X. F.; Luo, X. G.; Wang, A. F.; Zhang, M.; Yan, Y. J.; Xiang, Z. J.; Liu, R. H.; Cheng, P.; Ye, G. J. et al. Superconductivity and magnetic properties of single crystals of $\mathrm{K}_{0.75} \mathrm{Fe}_{1.66} \mathrm{Se}_{2}$ and $\mathrm{Cs}_{0.81} \mathrm{Fe}_{1.61} \mathrm{Se}_{2}$. Phys. Rev. B 2011, 83, 212502.

[3] Mou, D. X.; Zhao, L.; Zhou, X. J. Structural, magnetic and electronic properties of the iron-chalcogenide $\mathrm{A}_{x} \mathrm{Fe}_{2-y} \mathrm{Se}_{2}(\mathrm{~A}=\mathrm{K}, \mathrm{Cs}, \mathrm{Rb}$, and Tl, etc.) superconductors. Front. Phys. 2011, 6, 410-428.

[4] Dagotto, E. Colloquium: The unexpected properties of alkali metal iron selenide superconductors. Rev. Mod. Phys. 2013, 85, 849-867.

[5] Bao, W. Structure, magnetic order and excitations in the 245 family of Fe-based superconductors. J. Phys. Condens. Matter 2015, 27, 023201.

[6] Krzton-Maziopa, A.; Svitlyk, V.; Pomjakushina, E.; Puzniak, R.; Conder, K. Superconductivity in alkali metal intercalated iron selenides. J. Phys. Condens. Matter 2016, 28, 293002.

[7] Wu, M. K.; Wu, P. M.; Wen, Y. C.; Wang, M. J.; Lin, P. H.; Lee, W. C.; Chen, T. K.; Chang, C. C. An overview of the Fe-chalcogenide superconductors. J. Phys. D: Appl. Phys. 2015, 48, 323001.

[8] Bao, W.; Huang, Q. Z.; Chen, G. F.; Wang, D. M.; He, J. B.; Qiu, Y. M. A novel large moment antiferromagnetic order in $\mathrm{K}_{0.8} \mathrm{Fe}_{1.6} \mathrm{Se}_{2}$ superconductor. Chin. Phys. Lett. 2011, 28, 086104.

[9] Yan, Y. J.; Zhang, M.; Wang, A. F.; Ying, J. J.; Li, Z. Y.; Qin, W.; Luo, X. G.; Li, J. Q.; Hu, J. P.; Chen, X. H. Electronic and magnetic phase diagram in $\mathrm{K}_{x} \mathrm{Fe}_{2-y} \mathrm{Se}_{2}$ superconductors. Sci. Rep. 2012, $2,212$.

[10] Ding, X. X.; Fang, D. L.; Wang, Z. Y.; Yang, H.; Liu, J. Z.; Deng, Q.; Ma, G. B.; Meng, C.; Hu, Y. H.; Wen, H. H. Influence of microstructure on superconductivity in $\mathrm{K}_{x} \mathrm{Fe}_{2-y} \mathrm{Se}_{2}$ and evidence for a new parent phase $\mathrm{K}_{2} \mathrm{Fe}_{7} \mathrm{Se}_{8}$. Nat. Commun. 2013, 4, 1897.

[11] Zhang, Y.; Yang, L. X.; Xu, M.; Ye, Z. R.; Chen, F.; He, C.; Xu, H. C.; Jiang, J.; Xie, B. P.; Ying, J. J. et al. Nodeless superconducting gap in $\mathrm{A}_{x} \mathrm{Fe}_{2} \mathrm{Se}_{2}(\mathrm{~A}=\mathrm{K}, \mathrm{Cs})$ revealed by angle-resolved photoemission spectroscopy. Nat. Mater. 2011, 10, 273-277. 
[12] Qian, T.; Wang, X. P.; Jin, W. C.; Zhang, P.; Richard, P.; Xu, G.; Dai, X.; Fang, Z.; Guo, J. G.; Chen, X. L. et al. Absence of a holelike fermi surface for the iron-based $\mathrm{K}_{0.8} \mathrm{Fe}_{1.7} \mathrm{Se}_{2}$ superconductor revealed by angle-resolved photoemission spectroscopy. Phys. Rev. Lett. 2011, 106, 187001.

[13] Mou, D. X.; Liu, S. Y.; Jia, X. W.; He, J. F.; Peng, Y. Y.; Zhao, L.; Yu, L.; Liu, G. D.; He, S. L.; Dong, X. L. et al. Distinct fermi surface topology and nodeless superconducting gap in a $\left(\mathrm{Tl}_{0.58} \mathrm{Rb}_{0.42}\right) \mathrm{Fe}_{1.72} \mathrm{Se}_{2}$ superconductor. Phys. Rev. Lett. 2011, 106, 107001.

[14] Fang, M. H.; Wang, H. D.; Dong, C. H.; Li, Z. J.; Feng, C. M.; Chen, J.; Yuan, H. Q. Fe-based superconductivity with $T_{\mathrm{c}}=31 \mathrm{~K}$ bordering an antiferromagnetic insulator in $(\mathrm{Tl}, \mathrm{K}) \mathrm{Fe}_{x} \mathrm{Se}_{2}$. EPL 2011, 94, 27009.

[15] Tsurkan, V.; Deisenhofer, J.; Günther, A.; Krug von Nidda, H. A.; Widmann, S.; Loidl, A. Anisotropic magnetism, superconductivity, and the phase diagram of $\mathrm{Rb}_{1-x} \mathrm{Fe}_{2-y} \mathrm{Se}_{2}$. Phys. Rev. B 2011, 84, 144520.

[16] Ksenofontov, V.; Wortmann, G.; Medvedev, S. A.; Tsurkan, V.; Deisenhofer, J.; Loidl, A.; Felser, C. Phase separation in superconducting and antiferromagnetic $\mathrm{Rb}_{0.8} \mathrm{Fe}_{1.6} \mathrm{Se}_{2}$ probed by Mössbauer spectroscopy. Phys. Rev. B 2011, 84, 180508(R).

[17] Ricci, A.; Poccia, N.; Campi, G.; Joseph, B.; Arrighetti, G.; Barba, L.; Reynolds, M.; Burghammer, M.; Takeya, H.; Mizuguchi, Y. et al. Nanoscale phase separation in the iron chalcogenide superconductor $\mathrm{K}_{0.8} \mathrm{Fe}_{1.6} \mathrm{Se}_{2}$ as seen via scanning nanofocused X-ray diffraction. Phys. Rev. B 2011, 84, 060511.

[18] Charnukha, A.; Cvitkovic, A.; Prokscha, T.; Pröpper, D.; Ocelic, N.; Suter, A.; Salman, Z.; Morenzoni, E.; Deisenhofer, J.; Tsurkan, V. et al. Nanoscale layering of antiferromagnetic and superconducting phases in $\mathrm{Rb}_{2} \mathrm{Fe}_{4} \mathrm{Se}_{5}$ single crystals. Phys. Rev. Lett. 2012, 109, 017003.

[19] Li, W.; Ding, H.; Deng, P.; Chang, K.; Song, C. L.; He, K.; Wang, L. L.; Ma, X. C.; Hu, J. P.; Chen, X. et al. Phase separation and magnetic order in K-doped iron selenide superconductor. Nat. Phys. 2012, 8, 126-130.

[20] Carr, S. V.; Louca, D.; Siewenie, J.; Huang, Q.; Wang, A. F.; Chen, X. H.; Dai, P. C. Structure and composition of the superconducting phase in alkali iron selenide $\mathrm{K}_{y} \mathrm{Fe}_{1.6+x} \mathrm{Se}_{2}$. Phys. Rev. B 2014, 89, 134509.

[21] Speller, S. C.; Dudin, P.; Fitzgerald, S.; Hughes, G. M.; Kruska, K.; Britton, T. B.; Krzton-Maziopa, A.; Pomjakushina, E.; Conder, K.; Barinov, A. et al. High-resolution characterization of microstructural evolution in $\mathrm{Rb}_{x} \mathrm{Fe}_{2-y} \mathrm{Se}_{2}$ crystals on annealing. Phys. Rev. B 2014, 90, 024520 .

[22] Liu, Y.; Xing, Q. F.; Straszheim, W. E.; Marshman, J.; Pedersen, P.; McLaughlin, R.; Lograsso, T. A. Formation mechanism of superconducting phase and its three-dimensional architecture in pseudo-single-crystal $\mathrm{K}_{x} \mathrm{Fe}_{2-y} \mathrm{Se}_{2}$. Phys. Rev. B 2016, 93, 064509.

[23] Li, W.; Ding, H.; Li, Z.; Deng, P.; Chang, K.; He, K.; Ji, S. H.; Wang, L. L.; Ma, X. C.; Hu, J. P. et al. $\mathrm{KFe}_{2} \mathrm{Se}_{2}$ is the parent compound of K-doped iron selenide superconductors. Phys. Rev. Lett. 2012, 109, 057003.

[24] Zhao, J.; Cao, H. B.; Bourret-Courchesne, E.; Lee, D. H.; Birgeneau, R. J. Neutron-diffraction measurements of an antiferromagnetic semiconducting phase in the vicinity of the high-temperature superconducting state of $\mathrm{K}_{x} \mathrm{Fe}_{2-y} \mathrm{Se}_{2}$. Phys. Rev. Lett. 2012, 109, 267003.

[25] Zhou, Y.; Xu, D. H.; Zhang, F. C.; Chen, W. Q. Theory for superconductivity in $(\mathrm{Tl}, \mathrm{K}) \mathrm{Fe}_{x} \mathrm{Se}_{2}$ as a doped Mott insulator. EPL 2011, 95, 17003

[26] Cao, C.; Dai, J. H. Electronic structure and Mott localization of iron-deficient $\mathrm{TlFe}_{1.5} \mathrm{Se}_{2}$ with superstructures. Phys. Rev. B 2011, 83, 193104

[27] Yu, R.; Zhu, J. X.; Si, Q. M. Mott transition in modulated lattices and parent insulator of $(\mathrm{K}, \mathrm{Tl})_{y} \mathrm{Fe}_{x} \mathrm{Se}_{2}$ Superconductors. Phys. Rev. Lett. 2011, 106, 186401.

[28] Chen, F.; Xu, M.; Ge, Q. Q.; Zhang, Y.; Ye, Z. R.; Yang, L. X.; Jiang, J.; Xie, B. P.; Che, R. C.; Zhang, M. et al. Electronic identification of the parental phases and mesoscopic phase separation of $\mathrm{K}_{x} \mathrm{Fe}_{2-y} \mathrm{Se}_{2}$ superconductors. Phys. Rev. X 2011, 1, 021020.

[29] Xu, M.; Ge, Q. Q.; Peng, R.; Ye, Z. R.; Jiang, J.; Chen, F.; Shen, X. P.; Xie, B. P.; Zhang, Y.; Wang, A. F. et al. Evidence for an $s$-wave superconducting gap in $\mathrm{K}_{x} \mathrm{Fe}_{2-y} \mathrm{Se}_{2}$ from angle-resolved photoemission. Phys. Rev. B 2012, 85, 220504(R).

[30] Yi, M.; Lu, D. H.; Yu, R.; Riggs, S. C.; Chu, J. H.; Lv, B.; Liu, Z. K.; Lu, M.; Cui, Y. T.; Hashimoto, M. et al. Observation of temperatureinduced crossover to an orbital-selective mott phase in $\mathrm{A}_{x} \mathrm{Fe}_{2-y} \mathrm{Se}_{2}$ (A = K, Rb) superconductors. Phys. Rev. Lett. 2013, 110, 067003.

[31] Maletz, J.; Zabolotnyy, V. B.; Evtushinsky, D. V.; Yaresko, A. N.; Kordyuk, A. A.; Shermadini, Z.; Luetkens, H.; Sedlak, K.; Khasanov, R.; Amato, A. et al. Photoemission and muon spin relaxation spectroscopy of the iron-based $\mathrm{Rb}_{0.77} \mathrm{Fe}_{1.61} \mathrm{Se}_{2}$ superconductor: Crucial role of the cigar-shaped Fermi surface. Phys. Rev. B 2013, $88,134501$.

[32] Wang, X. P.; Qian, T.; Richard, P.; Zhang, P.; Dong, J.; Wang, H. D.; Dong, C. H.; Fang, M. H.; Ding, H. Strong nodeless pairing on separate electron Fermi surface sheets in $(\mathrm{Tl}, \mathrm{K}) \mathrm{Fe}_{1.78} \mathrm{Se}_{2}$ probed by ARPES. EPL 2011, 93, 57001

[33] Bendele, M.; Barinov, A.; Joseph, B.; Innocenti, D.; Iadecola, A.; Bianconi, A.; Takeya, H.; Mizuguchi, Y.; Takano, Y.; Noji, T. et al. Spectromicroscopy of electronic phase separation in $\mathrm{K}_{x} \mathrm{Fe}_{2-y} \mathrm{Se}_{2}$ superconductor. Sci. Rep. 2014, 4, 5592.

[34] Dudin, P.; Herriott, D.; Davies, T.; Krzton-Maziopa, A.; Pomjakushina, E.; Conder, K.; Cacho, C.; Yates, J. R.; Speller, S. C. Imaging the local electronic and magnetic properties of intrinsically phase separated $\mathrm{Rb}_{x} \mathrm{Fe}_{2-y} \mathrm{Se}_{2}$ superconductor using scanning microscopy techniques. Supercon. Sci. Technol. 2019, 32, 044005.

[35] Cao, C.; Dai, J. H. Block spin ground state and three-dimensionality of $(\mathrm{K}, \mathrm{Tl})_{y} \mathrm{Fe}_{1.6} \mathrm{Se}_{2}$. Phys. Rev. Lett. 2011, 107, 056401.

[36] Yan, X. W.; Gao, M.; Lu, Z. Y.; Xiang, T. Ternary iron selenide $\mathrm{K}_{0.8} \mathrm{Fe}_{1.6} \mathrm{Se}_{2}$ is an antiferromagnetic semiconductor. Phys. Rev. B 2011, 83, 233205.

[37] Chen, Z. G.; Yuan, R. H.; Dong, T.; Xu, G.; Shi, Y. G.; Zheng, P.; Luo, J. L.; Guo, J. G.; Chen, X. L.; Wang, N. L. Infrared spectrum and its implications for the electronic structure of the semiconducting iron selenide $\mathrm{K}_{0.83} \mathrm{Fe}_{1.53} \mathrm{Se}_{2}$. Phys. Rev. B 2011, 83, 220507(R). 